\title{
Fatty acid monolayers on randomly nanostructured inorganic surfaces: Interplay of wettability, chemistry and topography
}

\author{
M. Beauvais, I. Liascukiene, A. M. Jonas, J. Landoulsi
}

\section{Supporting information}

Number of pages: $11(\mathrm{~S} 1-\mathrm{S} 11)$

Number of Figures: 3 (Figure S1 - S3)

Number of Tables: 6 (Table S1 - S6)

\section{Content}

1. PSD/variogram calculations

2. Surface models

2.1. Models evaluated during the kriging step

2.2 Models for error evaluation

3. Wettability model

3.1. Dependency between variables analysis

3.2. Linear model assumptions evaluation and model evaluation

3.3. Variable importance 


\section{PSD/variogram calculations}

Table S1. $w_{s a t}, L_{c}$, sill and range parameters extracted from PSD and variogram calculations. Data are obtained from AFM images recorded on nanostructured Al surface modified with fatty acids.

\begin{tabular}{|c|c|c|c|c|}
\hline \multirow[t]{2}{*}{ Samples } & \multicolumn{2}{|c|}{ PSD calculation } & \multicolumn{2}{|c|}{ Variogram calculation } \\
\hline & $w_{\text {sat }}$ & $L_{c}$ & sill & range \\
\hline Al-nano_SA & 18.94 & 211.65 & 386.05 & 133.65 \\
\hline Al-nano_SA & 19.14 & 197.76 & 382.65 & 122.80 \\
\hline Al-nano_SA & 19.57 & 214.75 & 414.71 & 139.07 \\
\hline A-nano_OA & 17.68 & 181.49 & 312.18 & 117.99 \\
\hline Al-nano_OA & 17.03 & 177.15 & 291.65 & 119.02 \\
\hline Al-nano_LA & 15.77 & 176.58 & 263.30 & 119.75 \\
\hline Al-nano_LA & 16.49 & 176.82 & 289.33 & 125.43 \\
\hline Al-nano_LA & 16.73 & 178.48 & 295.79 & 125.27 \\
\hline Al-nano_LA & 15.40 & 175.30 & 255.34 & 110.87 \\
\hline Al-nano_LrA & 23.69 & 239.92 & 609.56 & 152.95 \\
\hline Al-nano_CA & 18.44 & 170.98 & 343.81 & 110.01 \\
\hline Al-nano_CA & 17.40 & 159.73 & 314.03 & 103.36 \\
\hline Al-nano_OA* & 16.04 & 174.19 & 274.39 & 118.63 \\
\hline Al-nano_OA* & 16.24 & 174.28 & 278.84 & 116.08 \\
\hline Al-nano_OA* & 16.48 & 173.23 & 290.10 & 122.79 \\
\hline Al-nano_OA* & 16.87 & 184.42 & 299.29 & 124.42 \\
\hline
\end{tabular}

*further incubated in air for $24 \mathrm{~h}$ at room temperature 


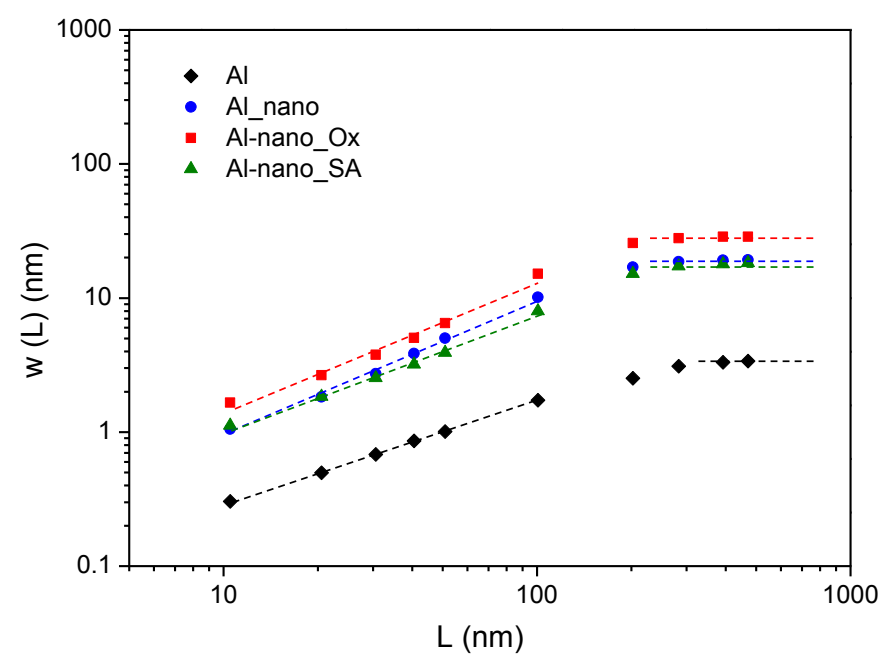

Figure S1. Log-log plots showing the evolution of the root-mean-square roughness, $w(L)$, as a function of the length scale, $L$. The plots are constructed from PSD analysis of AFM images recorded on aluminum substrate prior to $(A l)$ or after hydroxylation treatment (Al-nano) and further incubation in hydrogen peroxide solution (Al-nano_Ox), or on Al-nano surface modified with stearic acid (Al-nano_SA). Dashed lines indicate the roughness changes predicted by Eq. (3) and the saturation at large length scales. The fitting at small length scales with a power law yields roughness exponent values: Al: 0.77; Al-nano: 1.02;Al-nano_Ox: 0.98;Al-nano_SA: 0.86 


\section{Surface models}

\subsection{Models evaluated during the kriging step}

18 models were evaluated and the Pentaspherical model, giving the best fit, was selected to model each surface. The range was extracted from the Pentaspherical model calculated from the experimental variogram as following.

$\operatorname{pen}(\mathrm{d})=\frac{15 d}{8 a}-\frac{5}{4}\left(\frac{d}{a}\right)^{3}+\frac{3}{8}\left(\frac{d}{a}\right)^{5}, \quad 0<d<a$

$\operatorname{pen}(\mathrm{d})=1, \quad \mathrm{~d}>a$

where $a$ is the range.

\subsection{Models for error evaluation}

The surface models were evaluated with both correlation and precision.

The correlation is Person's correlation between the predicted heights and heights of the surface.

The precision is defined as the sum over the surface of the square of the difference of heights (the difference between one height of the surface and the corresponding predicted height).

Table S2. Pearson's correlation between heights and predicted heights of the surface and precision of the height's prediction

\begin{tabular}{llll}
\hline Samples & Model & correlation & precision \\
\hline Al-nano_SA & Nug. Pen & 0.99 & 1251529.89 \\
Al-nano_SA & Nug. Pen & 0.99 & 1462709.58 \\
Al-nano_SA & Nug. Pen & 0.99 & 1284667.28 \\
Al-nano_OA & Nug. Pen & 0.99 & 1747867.96 \\
Al-nano_OA & Nug. Pen & 0.99 & 1134987.80 \\
Al-nano_LA & Nug. Pen & 0.98 & 2015266.04 \\
Al-nano_LA & Nug. Pen & 0.99 & 1938286.76 \\
Al-nano_LA & Nug. Pen & 0.99 & 2052573.79 \\
Al-nano_LA & Nug. Pen & 0.98 & 2084821.49 \\
Al-nano_LrA & Nug. Pen & 1.00 & 1203891.54 \\
Al-nano_CA & Nug. Pen & 0.99 & 1941571.48 \\
Al-nano_CA & Nug. Pen & 0.99 & 1838151.23 \\
Al-nano_OA* & Nug. Pen & 0.99 & 1577481.86 \\
Al-nano_OA* & Nug. Pen & 0.99 & 1467583.43 \\
Al-nano_OA* & Nug. Pen & 0.99 & 1437504.98 \\
Al-nano_OA* & Nug. Pen & 0.99 & 1744896.98
\end{tabular}

* further incubated in air for $24 \mathrm{~h}$ at room temperature 


\section{Wettability model}

\subsection{Dependency between variables analysis}

Multiple linear regression was used to examine the impacts of $L_{c}, w_{\text {sat }}$, sill and range variables on the variations of $\theta_{\mathrm{w}}$. This method requires that variables and model obey to two main conditions. First, variables must be independent. Figure S2 shows that several pairs of variables are highly correlated. range and sill were, thus, considered in the modeling as independent variables of topography, and $A_{v(C-H)}$ for chemistry. Second, the four assumptions of linear model should be validated: (i) linearity and additivity of the relationship between dependent and independent variables, (ii) statistical independence of the errors, (iii) homoscedasticity (constant variance) of the errors and (iv) normality of the error distribution.

These conditions require the transformation of variables to make them following a Normal or nearly Normal distribution. Several transformations including Box-Cox transformation [S5] among others were tested and evaluated for each variable and the transformed variable with the best Pearson P statistic divided by its degrees of freedom is retained. The $\mathrm{A}_{v(\mathrm{C}-\mathrm{H})}$ variable was transformed with the $\log \left(10 \times A_{v(C-H)}\right)$ function, while both range and sill factors were transformed with a Tukey's Ladder of Powers). range was transformed with the $-1 *$ range $^{-2.15}$ function and sill with the $-1 *$ sill $^{-3.85}$ function.

In order to perform the wettability prediction modeling, the dependency between variables was evaluated using the cor.test function of the stats R package to calculate the Pearson's productmoment correlation. The chemistry related variable $A_{v(C-H)}$ was clearly assessed with Figure S2 to be not linearly dependent of the randomness of topography variable (range). 


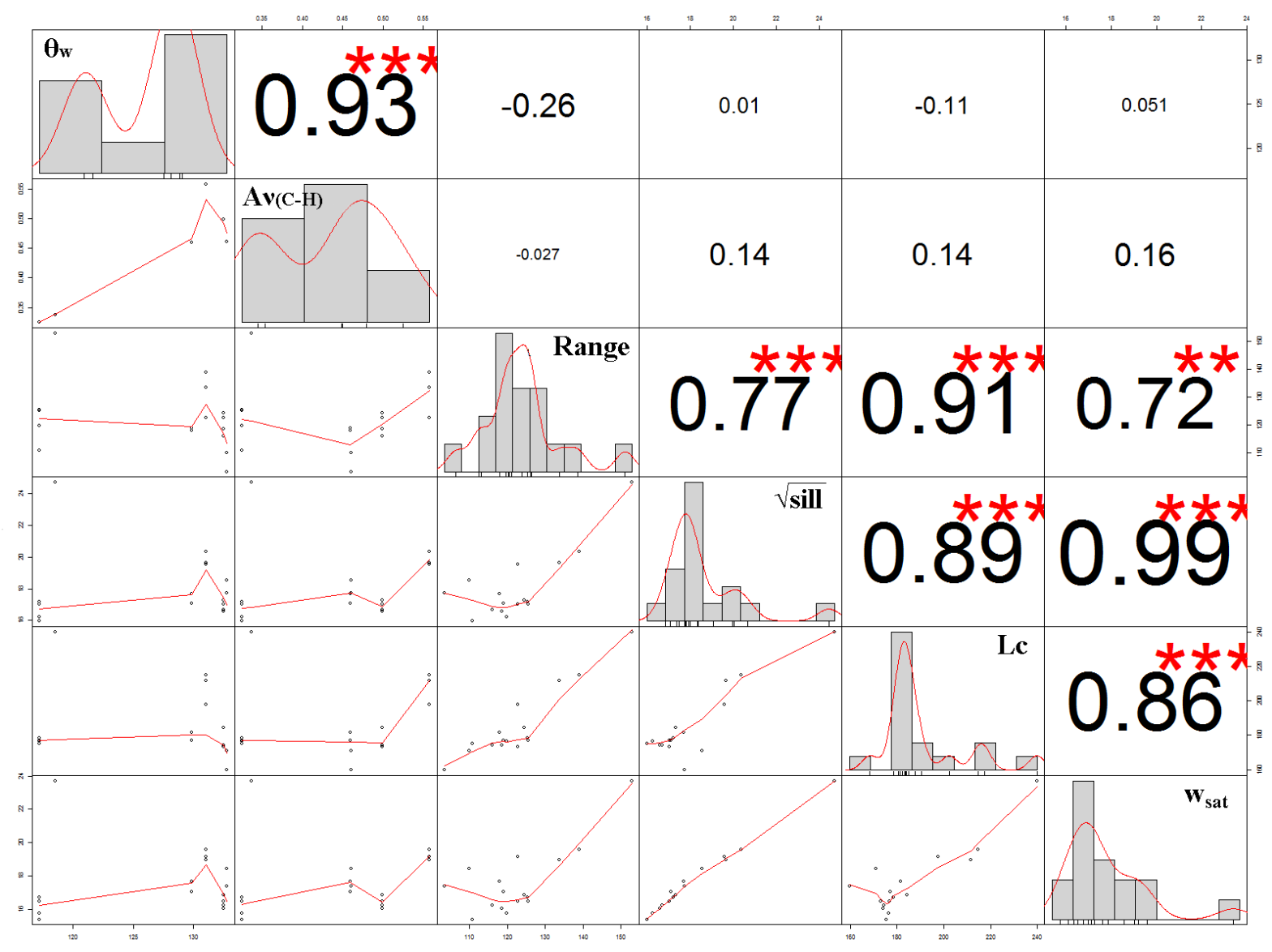

Figure S2. Correlation matrix. On top are the absolute values of the correlation and the result of the Pearson's product moment correlation coefficient between paired samples as stars. On bottom, the bivariate scatterplots, with a fitted line. On the diagonal, distribution of values and probability density function.

\subsection{Linear model assumptions evaluation and model evaluation}

Each possible model, as a simple combination of independent variables, was also evaluated with the Akaike information criterion (AIC) with the stepwise algorithm implemented in the MASS R package [S6] and used in Code S1.

Code S1: Model selection with the Akaike information criterion (AIC) with a stepwise algorithm The data from the [table S1] table is stored in the group2 variable library(MASS) modlin $=\operatorname{lm}($ WCA $\sim$, data $=$ group 2$)$

summary(modlin) napp=length (group2\$WCA) modselect_AIC=stepAIC(modlin, , ,trace=TRUE, scope $=$ list $($ upper $=\sim .$, lower $=\sim 1)$, direction=c("both"), $\mathrm{k}=\log ($ napp)) 
summary(modselect_AIC)

modselect_AIC

The model with the best AIC was

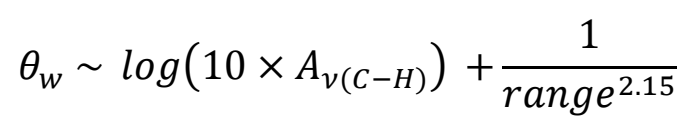

A simple linear model calculation confirmed that the wettability is well explained by the two transformed variables $\mathrm{A}_{v(\mathrm{C}-\mathrm{H})}$ and range (Table S3).

Table S3. Characteristics of the linear model

\begin{tabular}{l|llll}
\hline & Estimate & Std. Error & $\mathrm{t}$ value & $\operatorname{Pr}(>|\mathrm{t}|)$ \\
\hline Intercept & 74.04 & 4.13 & 17.91 & $1.52 \mathrm{e}-10$ \\
$\log \left(10 \times A_{\nu(C-H)}\right)$ & 69.91 & 13.60 & 13.60 & $4.60 \mathrm{e}-09$ \\
Tukey range & -252200 & 74630 & -3.38 & 0.00493 \\
\hline adjusted $\boldsymbol{R}^{2}$ & $\mathbf{0 . 9 2 8 6}$ & & & \\
p-value & $\mathbf{1 . 3 9 5 e - 8}$ & & & \\
\hline
\end{tabular}

The linear model assumptions were successfully validated visually with Figure S3.
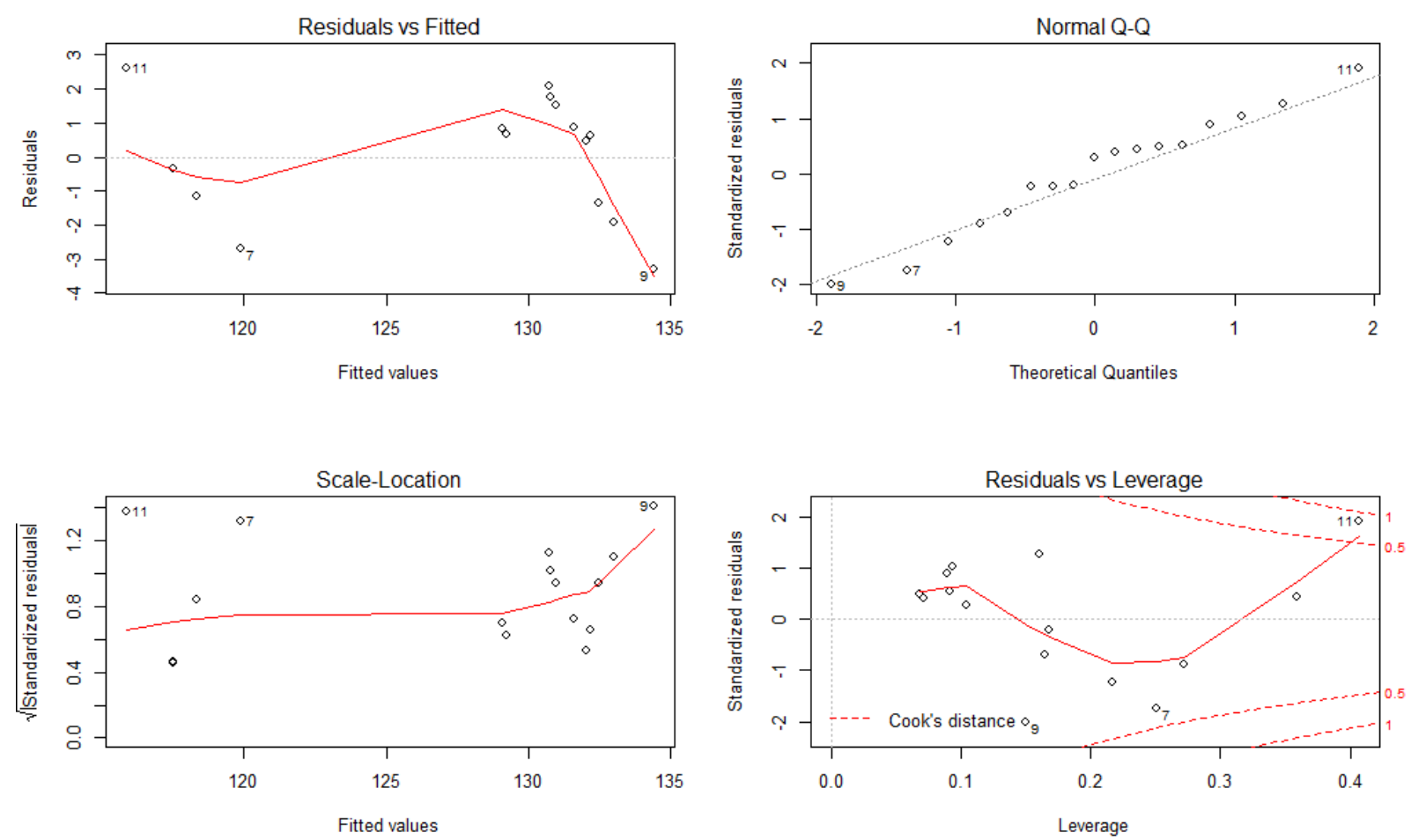

Figure S3. Key visual tests of the linear model. 
The linear model assumptions were successfully globally validated with a global method [S3] implemented in the gvlma method of the gvlma R package [S4] and used in Code S2.

Code S2: Global validation of linear model assumptions

library(gvlma)

$\operatorname{summary}(\operatorname{gvlma}(\mathrm{x}=$ modselect_AIC, alphalevel $=0.05))$

\subsection{Variable importance}

The variable importance (Table S4), the absolute value of the $t$ value for each model parameter, was calculated using the varImp method in the R caret package [S2] and used in Code S3. The Tukey transformed range factor is four times less important than the $\log \left(10 \times A_{v(C-H)}\right)$ factor in the prediction of WCA.

Table S4. Variables importance

\begin{tabular}{cc}
\hline Tukey range & 3.38 \\
\hline $\boldsymbol{l o g}\left(\mathbf{1 0} \times \boldsymbol{A}_{\boldsymbol{v}(\boldsymbol{C}-\boldsymbol{H})}\right)$ & 13.59 \\
\hline
\end{tabular}

\section{Code S3: Variable importance evaluation}

library(caret)

varImp (modselect_AIC, scale $=$ FALSE) 
Table S5. Parameters used in the wettability model and comparison between experimental and predicted $\theta_{w}$ values.

\begin{tabular}{llllll}
\hline Sample & $\mathbf{A}_{\boldsymbol{v}(\mathrm{C}-\mathrm{H})}$ & Range & $\theta_{w}$ & predicted $\theta_{w}$ & Error (\%) \\
\hline Al-nano_SA & 0.558 & 133.6476 & 131.1 & 133.0138 & 1.45981262 \\
Al-nano_SA & 0.558 & 122.7969 & 131.1 & 134.3669 & 2.491927231 \\
Al-nano_SA & 0.558 & 139.0725 & 131.1 & 132.4582 & 1.036015805 \\
Al-nano_OA & 0.46 & 117.9916 & 129.9 & 129.2318 & 0.51438745 \\
Al-nano_OA & 0.46 & 119.0183 & 129.9 & 129.0683 & 0.640228937 \\
Al-nano_LA & 0.325 & 119.748 & 117.2 & 118.4075 & 1.030266236 \\
Al-nano_LA & 0.325 & 125.4332 & 117.2 & 117.5930 & 0.335327148 \\
Al-nano_LA & 0.325 & 125.2678 & 117.2 & 117.6151 & 0.354153957 \\
Al-nano_LA & 0.325 & 110.8662 & 117.2 & 119.9539 & 2.349722253 \\
Al-nano_LrA & 0.337 & 152.9534 & 118.5 & 115.9973 & 2.11200259 \\
Al-nano_CA & 0.461 & 110.0119 & 132.8 & 130.7370 & 1.553468683 \\
Al-nano_CA & 0.461 & 103.3646 & 132.8 & 132.2136 & 0.441579382 \\
Al-nano_OA* & 0.499 & 118.6257 & 132.5 & 131.6011 & 0.678446309 \\
Al-nano_OA* & 0.499 & 116.0797 & 132.5 & 132.0192 & 0.362859243 \\
Al-nano_OA* & 0.499 & 122.7864 & 132.5 & 130.9755 & 1.150549488 \\
Al-nano_OA* & 0.499 & 124.4191 & 132.5 & 130.7478 & 1.322388314 \\
\hline
\end{tabular}

*further incubated in air for $24 \mathrm{~h}$ at room temperature 
Table S6. Wenzel roughness computed from the AFM images, and computed water contact angle if the surface be flat.

\begin{tabular}{lcc}
\hline Sample & Wenzel roughness, $\boldsymbol{r}$ & $\theta_{f}\left({ }^{\circ}\right)$ \\
\hline Al-nano_SA & 1.52 & 116 \\
Al-nano_SA & 1.51 & 116 \\
Al-nano_SA & 1.5 & 116 \\
Al-nano_OA & 1.43 & 117 \\
Al-nano_OA & 1.48 & 116 \\
Al-nano_LA & 1.44 & 109 \\
Al-nano_LA & 1.48 & 108 \\
Al-nano_LA & 1.5 & 108 \\
Al-nano_LA & 1.51 & 108 \\
Al-nano_LrA & 1.41 & 110 \\
Al-nano_CA & 1.46 & 118 \\
Al-nano_CA & 1.46 & 118 \\
Al-nano_OA* & 1.47 & 117 \\
Al-nano_OA* & 1.47 & 117 \\
Al-nano_OA* & 1.55 & 116 \\
Al-nano_OA* & 1.55 & 116 \\
\hline
\end{tabular}

* further incubated in air for $24 \mathrm{~h}$ at room temperature 


\section{References}

[S1] gstat user's manual, EJ Pebesma - 1992 - gstat.org

[S2] https://cran.r-project.org/web/packages/caret/index.html

[S3] Pena, EA and Slate, EH (2006). "Global validation of linear model assumptions," J. Amer. Statist. Assoc., 101(473):341-354.

[S4] https://cran.r-project.org/web/packages/gvlma/index.html

[S5] (a) Box, G. E. P. and Cox, D. R. (1964) An analysis of transformations, Journal of the Royal Statistical Society B, 26, 211-252. (b) Tukey's Ladder of Powers (Tukey, J. (1977). Exploratory Data Analysis. Addison-Wesley). (c)Yeo-Johnson transformation (A New Family of Power Transformations to Improve Normality or Symmetry, In-Kwon Yeo and Richard A. Johnson - Biometrika Vol. 87, No. 4 (Dec., 2000), pp. 954-959). (d) Ordered Quantile technique (Order Tests for the Two-Sample Problem and their Power - B.L.Van Der Waerden Indagationes Mathematicae (Proceedings) Volume 56, 1953, Page 80).

[S6] https://cran.r-project.org/web/packages/MASS/index.html 